\title{
Estudiantes universitarios indígenas y procesos formativos
}

\section{Indigenous university students and training processes}

\author{
Cecilia Navia Antezana* \\ GABRIELA CZARNY** \\ Gisela Victoria Salinas SÁnchez ${ }^{* * *}$
}

El objetivo de este artículo es analizar los procesos formativos de los estudiantes de la licenciatura en Educación Indígena de la Universidad Pedagógica Nacional. Desde un enfoque interpretativo, utilizamos dos estrategias de recuperación de información: un cuestionario de preguntas abiertas y registros de trabajo de acompañamiento pedagógico. El texto se inscribe en el campo de la educación superior y los pueblos indígenas, que en los últimos años se han venido visibilizando en el debate sobre las formas de producir conocimiento y la importancia de recuperar las voces silenciadas en los procesos de profesionalización indígena. Estas exigencias se dan en el marco de la confrontación de discursos del neoindigenismo de Estado, las perspectivas poscoloniales y decoloniales, y del papel de la academia y los pueblos indígenas en los procesos de producción de conocimiento. La experiencia universitaria ofrece elementos que aportan a la reconfiguración personal y colectiva de sus procesos de formación y adscripción comunitaria, y la revalorización de sí mismos como sujetos individuales y colectivos, espacios de interacción que favorecen disposiciones para desarrollar proyectos para y con los pueblos indígenas.

The objective of this article is to analyze the training processes of students of the licenciatura en Educación Indígena de la Universidad Pedagógica Nacional. From an interpretative perspective were use two strategies to gather information: An opened questionnaire and accompany pedagogy work records. The text comes within the field of higher education and indigenous people that in the last years have been visibilized in the debate about the ways of producing knowledge and the importance to reclaim the silence voices in the processes of the indigenous professionalization. These exigencies are shown in the confrontation field of speeches in neo-indigenism's State, from the postcolonial and de-colonial perspective and the roll of academy and indigenous people in the knowledge processes production. The university experience offers elements that give to the personal and collective reconfiguration to their training processes and community ascription, to strength themselves as individual and collective subject, in interaction that contains provisions in favor to develop projects for and with indigenous people.

\section{Palabras clave:}

educación superior, estudiantes indígenas, formación de profesionales, dedescolonización

\section{Keywords:}

higher education, indigenous students, professional training dedescolonization

Recibido: 2 de septiembre de 2018. | Aceptado para su publicación: 7 de enero de 2019. Recuperado de: https://sinectica.iteso.mx/index.php/SINECTICA/article/view/912 DOI: 10.31391/S2007-7033(2019)0052-002

\footnotetext{
* Doctora en Ciencias de la Educación por la Universidad Sorbona París 3. Docente e investigadora en la Universidad Pedagógica Nacional, Unidad Ajusco. Área académica Diversidad e Interculturalidad. Líneas de investigación: Formación docente, identidad y ética profesional. Correo electrónico: ceeeci@yahoo.com/https://orcid.org/0000-0001-6721-8205

** Doctora en Ciencias con especialidad en Investigaciones Educativas. Docente e investigadora en la Universidad Pedagógica Nacional, Unidad Ajusco. Área académica Diversidad e Interculturalidad. Líneas de investigación: escolarización indígena en contextos urbanos y de migración, jóvenes indígenas y educación superior, y formación docente para contextos de diversidad sociocultural. Correo electrónico: gacza_2006@yahoo.com.mx/https://orcid.org/0000-0002-7638-4229

${ }^{* * *}$ Doctora en Desarrollo Educativo con Énfasis en Formación de Docente. Docente e investigadora en la Universidad Pedagógica Nacional, Unidad Ajusco. Área académica Diversidad e Interculturalidad. Líneas de investigación: formación de docentes para la educación indígena e intercultural. Correo electrónico: gsalinas@upn.mx/https://orcid. org/0000-0001-9069-6942
} 


\section{INTRODUCCIÓN}

$\mathrm{E}$ I trabajo se inscribe en el debate de la educación superior y los pueblos indígenas, particularmente en el debate que en los últimos treinta años ha adquirido visibilidad en América del Sur y Centroamérica, en el que se cuestionan los modos de producir conocimiento y la unívoca noción de ciencia como un universal, procesos marcados por la raíz de pensamiento eurocéntrico. En este escenario, las perspectivas conocidas como interculturalismo crítico (Tubino, 2007; Prada y López, 2009) y el debate poscolonial y decolonial (Mignolo, 2010; Restrepo y Rojas, 2010; Walsh, 2009) han abierto intersticios no solo para que se escuchen las voces silenciadas de los pueblos indígenas, sino también para que la academia, las instituciones y los conceptos que las han determinado como discursos únicos se comiencen a "cuestionar".

En México, el campo de lo que se conoce como profesionalización indígena tiene una historia propia en la que se confrontan los discursos del indigenismo de Estado, la participación de los académicos comprometidos y de los mismos profesionales y académicos indígenas. En este sentido, desentrañar las superposiciones, contradicciones y juegos de poder en este ámbito resulta una tarea aún por recuperar.

Como parte del proyecto "Formadores de profesionales de y para la educación indígena. Retos para una descolonización académica" de la Universidad Pedagógica Nacional (UPN) Ajusco, analizamos en este artículo los procesos formativos de estudiantes de la licenciatura en Educación Indígena (LEI), a partir de la recuperación de las respuestas a un cuestionario de seguimiento y evaluación sobre el programa educativo, así como de los registros de trabajo docente que las autoras realizamos con base en el acompañamiento del proceso formativo para la titulación.

Encontramos que la licenciatura les ofrece a los estudiantes elementos que aportan a la reconfiguración personal y colectiva de sus procesos de formación y de adscripción comunitaria, la revaloración de sí mismos como sujetos individuales y colectivos, y el reconocimiento de procesos formativos que se producen a partir de la interacción con los formadores, con sus pares y con la institución que generan disposiciones favorables a la intervención y el desarrollo de proyectos educativos en sus comunidades.

En los siguientes apartados presentamos una descripción general de la licenciatura, los referentes teóricos y perspectiva metodológica desde la que partimos para analizar la problemática; finalmente, abordamos la reconfiguración personal y colectiva en y de los procesos formativos con base en la formación y adscripción a las comunidades, la revaloración de sí mismos como sujetos individuales y colectivos, además de las tensiones en la formación y las posibilidad de los proyectos educativos a futuro y su relación con las comunidades y los pueblos indígenas.

\section{CARACTERÍSTICAS DEL PROGRAMA EDUCATIVO EN LA UNIVERSIDAD Pedagógica nacional, Ajusco}

La LEI, como programa educativo, opera desde 1982 en la UPN Ajusco y tiene como meta la formación de profesionales indígenas que contribuyan al campo de la educación para los pueblos indígenas. Durante casi dos décadas, la LEI atendió solo a maestros y directivos de educación indígena que no contaban con estudios de licenciatura y que se encontraban trabajando, principalmente en los niveles de preescolar y primaria. 
Estos maestros, hablantes de diversas lenguas originarias y provenientes de distintas comunidades indígenas, habían pasado por lo que se conoce como cursos de inducción a la docencia indígena impartidos por la Dirección General de Educación Indígena, de la Secretaría de Educación Pública; sin embargo, no contaban con una formación en educación superior que les permitiera contar con diversas herramientas para este campo. En este sentido, el programa se orientó a formar profesionales técnico-pedagógicos del campo educativo que, desde una perspectiva de los propios docentes indígenas y una preparación universitaria, pudieran contribuir a diseñar proyectos y estrategias descolonizadoras que les ayudaran a decidir la educación de los pueblos (Salinas, 2011).

Desde el año 2000, la LEI ha experimentado un cambio en el perfil de ingreso de los estudiantes, ya que comienzan a llegar sobre todo jóvenes bachilleres provenientes de entidades con distintos niveles de vinculación hacia sus comunidades y con situaciones diversas en el dominio y uso de las lenguas originarias. Estos jóvenes, al igual que muchos otros no indígenas, llegan con expectativas diversas a la universidad. Asimismo, un sector del estudiantado de la LEI proviene de comunidades de algunas entidades federativas, mientras que otros ya radican desde pequeños en la Ciudad de México o en su zona conurbada. Para los primeros, el cambio de residencia implica importantes procesos de reacomodo personal en los cuales los lazos con familiares y amigos resultan centrales para afrontar choques socioculturales, discriminaciones, así como el habitus que la academia tiene frente a los estudiantes indígenas.

Este programa resulta para los estudiantes un desafío en relación con su futuro profesional, ya sea para incorporarse a la docencia, a diferentes instituciones e instancia educativas. Para la UPN, ofrecer estudios de nivel superior a docentes y directivos de educación indígena, y en los últimos años a jóvenes bachilleres, ha implicado un fuerte reto para los formadores por la complejidad de atender una educación planteada para los pueblos y, más recientemente, por los pueblos indígenas.

Esto ha exigido a los académicos que se han involucrado a lo largo de los años en este programa educativo cuestiones como el desarrollo de investigaciones en escuelas y comunidades indígenas, la participación en diferentes procesos de formación y actualización de docentes y formadores de docentes indígenas en servicio, así como su incorporación en proyectos educativos que, en los últimos años, comienzan a plantearse e impulsarse desde las mismas comunidades.

A través de la autorreflexión del colegiado de la licenciatura, se advierte que en las prácticas de formación heterogéneas, que desde su creación se han desarrollado por maestros y estudiantes, están presentes, de cierta manera, formas de indianización de las aulas de la UPN Ajusco. Los espacios formativos de la LEI son experiencias únicas de diálogo, no carentes de tensión, que se establecen entre sujetos de una gran diversidad sociocultural y lingüística, así como con vinculaciones etnopolíticas diversas. Sin embargo, las marcas de lo étnico en este programa de estudios, desde su nombre, así como en las prácticas diferenciadas -por ejemplo, para el ingreso mediante un examen diferente al que realizan los aspirantes de otras carreras que ofrece la UPN-, nos llevan a interrogarnos si en algunos casos pueden resultar, en cierto nivel, un "estigma".

Al igual que en la mayoría de los actuales programas que se ofrecen en educación superior para la población indígena, las discriminaciones sobre los pueblos siguen marcando la división entre indios y no indios, aspecto que en otros niveles también 
carga con las desiguales condiciones, tanto académicas como económicas, con las que muchos de los jóvenes llegan a la universidad (Czarny, 2012).

En educación superior ha sido una constante el poco acceso a estudiantes procedentes de diferentes grupos indígenas del país, lo cual diversos autores han considerado como un proceso de exclusión hacia este sector de la población. De hecho, no hay una cifra clara, y se estima que entre el 1\% y el 3\% de los estudiantes de educación superior son indígenas (Villa, 2018). Existen pocas investigaciones que dan cuenta de las trayectorias de los estudiantes, el rezago y deserción, así como acerca del porcentaje de estudiantes que han logrado egresar y titularse de programas universitarios. De igual modo, poco se sabe de cómo los procesos formativos han incidido en las reconfiguraciones personal y colectiva de los estudiantes y en relación con su vínculo con las comunidades.

\section{REFERENTES TEÓRICOS}

Retomamos como referentes teóricos la discusión sobre descolonización en la educación superior, en particular lo relativo al papel que la educación superior juega en la formación de jóvenes indígenas. En primer lugar y de acuerdo con Restrepo y Rojas (2010), “con descolonización se indica un proceso de superación del colonialismo, generalmente asociado a las luchas anticoloniales en el marco de estados concretos. La decolonialidad, en cambio, refiere al proceso que busca trascender históricamente la colonialidad" (p. 16).

Sin embargo, las historias locales actúan de distinto modo entre estos dos grandes procesos mencionados. Por ello, hay que reconocer que las rupturas no son completas cuando se habla de descolonizar y decolonialidad en la educación superior, y que en el contexto de resistencia latinoamericanos, la educación intercultural crítica ha representado, tal vez, una vereda alterna a los discursos de opresión y racialización. En este aspecto, gran parte de los planteamientos de educación superior han retomado la perspectiva intercultural, aunque desde diversos significados.

Al respecto, Tubino (2007) destaca la apertura de espacios sociales formativos en las universidades siempre y cuando permitan responder a problemas de injusticia cultural y asimetría social. Por su parte, Prada y López (2009) resaltan la importancia de reconsiderar planteamientos epistemológicos al abordar los procesos formativos. Ello refiere la búsqueda por replantear el modelo metodológico y conceptual universal que rige lo que se ha denominado construcción de conocimiento científico.

Mato (2017) ha trabajado el campo de la educación superior y los pueblos indígenas en perspectivas comparativas en la región latinoamericana, y subraya la relevancia de analizar las posturas colaborativas al hacer frente en la educación superior a "siglos de racismo y mentalidades coloniales" (p. 17). Resalta la necesidad de hacerlo de manera académicamente informada y, a la vez, con sentido práctico. Cuestiona, en cierto nivel, lo que se viene desarrollando en el campo de estudios con base en la noción de diálogo de saberes, y señala que las posibilidades para ese diálogo no siempre se producen en condiciones de equidad, por lo que apuesta hacia procesos a los que llama "hacer juntos"; entiende por ello las dinámicas de encuentros y desencuentros que contiene el acto pedagógico en el marco de tensiones institucionales de diversos tipos. 
También, al analizar procesos en universidades interculturales, y desde una búsqueda por un trabajo colaborativo, Dietz y Mateos (2010) proponen una etnografía doblemente reflexiva que, al ubicar la estructura del poder, contribuya “a acompañar a los actores en sus itinerarios de movilización y reivindicación discursiva, pero también de interacción vivencial y de transformación práctica, que los sitúa de forma muy heterogénea entre culturas, entre saberes y entre poderes" (p. 128).

Estas perspectivas entran más bien, desde el terreno concreto de trabajo en instituciones con jóvenes indígenas, en un diálogo con un horizonte decolonial (o descolonizante) que, retomando a Mignolo (2010), apuntan a "develar los silencios epistémicos de la epistemología occidental y afirmar los derechos epistémicos de las opciones decoloniales racialmente devaluadas, para permitir, desde el silencio, construir argumentos que confronten a aquéllos que toman a la 'originalidad' como el criterio máximo para el juicio final" (p.13).

Los resultados que presentamos también tienen como referente de análisis la teoría de la agencia de Arendt (2001), en particular la importancia que la autora otorga a la palabra y la narración como acción, a partir de la cual es posible la reflexión de la experiencia y, con esta acción, es posible acceder al mundo. Por otro lado, se recuperan las teorías de la formación y el acompañamiento de Navia (2006) y Magnan (1997), quienes colocan en el centro del proceso formativo al estudiante o al sujeto en formación.

\section{ENFOQUE METODOLÓGICO E INTERPRETATIVO}

La investigación se desarrolló desde un enfoque hermenéutico comprensivo. Recupera a Ricoeur (2001) en la perspectiva de que los discursos pueden ser considerados a las vez como acciones en el sentido de que, por su carácter referencial, están vinculados de manera significativa a los sujetos de estudio, considerando que es del mundo de lo que se habla y, por ello, "el discurso significa plenamente" (p. 130). A partir del análisis de los significados y sentidos que los estudiantes otorgan a los procesos formativos al ser estudiantes de educación superior en la LEI, intentamos recuperar el carácter significativo de su experiencia.

Se trata de reconstruir las tramas de significación que enmarcan las prácticas académicas y socioculturales en las que los estudiantes se inscriben. Desde esta perspectiva, incorporamos la inserción de estas tramas considerando el marco histórico y cultural en el cual se desarrollan los procesos formativos de los estudiantes de la licenciatura. En un contexto de globalización que intenta imponer un proceso de homogeneización privilegiando un modo de ver y vivir el mundo con una perspectiva occidental, es relevante recuperar la voz de los sujetos desde una perspectiva epistemológica que busca horizontalidad a través de una relación de sujeto-sujeto orientada a conocer conjuntamente.

De ahí que este trabajo tiene una perspectiva metodológica cualitativa con carácter interpretativo y crítico, que coincide con Vasilachis (2007) en que, en el desarrollo de la investigación, no se pueden aislar los presupuestos de orden filosófico, ético, teórico y político presentes en los diferentes momentos del trabajo. De esta manera, asumimos una postura que considere un diálogo colaborativo entre quienes estamos implicados (Suárez, 2010), ya que somos docentes, tutores y asesores de trabajos de 
titulación de estudiantes del programa educativo en cuestión, lo que nos ha interpelado, a lo largo del acompañamiento a los estudiantes, en el seguimiento y la evaluación de sus procesos formativos.

En este sentido, integramos como una herramienta analítica la autorreflexión y la reflexión entre pares, siguiendo la perspectiva de Suárez (2010), definida como "espacios de trabajo colaborativo que se orientan a desarrollar y a poner a prueba formas de nombrar y considerar en términos pedagógicos 'lo que sucede' en los espacios escolares y 'lo que les sucede' a los actores educativos cuando los hacen y transitan" (p. 72).

Recuperando estos referentes metodológicos, se siguieron dos estrategias para recopilar la información: un cuestionario de preguntas abiertas, dirigido a estudiantes, y registros de nuestras sesiones de asesoría y acompañamiento para la elaboración de trabajos de titulación.

El cuestionario de seguimiento y evaluación para estudiantes de la LEI tuvo como propósito conocer la percepción que ellos tienen sobre algunos aspectos que componen el programa educativo, Plan 2011, y sobre su experiencia personal y estudiantil durante la formación profesional en la licenciatura y en la UPN. Los estudiantes tuvieron conocimiento de la intención del cuestionario, que respondía al proceso de seguimiento y evaluación de la licenciatura, y se les explicó que su llenado era voluntario, y no obligatorio. Para garantizar el anonimato no se incorporó el nombre de los estudiantes en el instrumento.

Los datos obtenidos corresponden a una muestra voluntaria (Sampieri, 2006) de estudiantes que contestaron el cuestionario. Consideramos como características de la población: que fueran estudiantes en la licenciatura, que se encontraran cursando el octavo semestre, que es el último del programa, o que estuvieran cursando otros semestres atendidos por un tutor del sistema que ofrece el Centro de Atención de Estudiantes. De este modo, la población total de estudiantes en el momento de la aplicación del instrumento fue de 203 estudiantes. Los cuestionarios se entregaron a los asesores de los estudiantes y directamente a los estudiantes durante los espacios de asesoría y los cursos de seminarios del octavo semestre, así como en reuniones de comités lectores de los trabajos de titulación, entre marzo y mayo de 2017. Los estudiantes recibieron el cuestionario de forma impresa y los interesados respondieron a mano; en tres casos, estos enviaron las respuestas en formato digital.

El cuestionario se integró de cuatro preguntas abiertas: ¿cuáles eran tus expectativas formativas al ingresar en la LEI-UPN y cómo son ahora?; ¿cómo ha incidido tu formación en la LEI para fortalecer diversos procesos culturales, lingüísticos, identitarios, así como la posibilidad de participar en proyectos en los que puedes desarrollar tus habilidades profesionales (en la comunidad, en distintas instituciones, etcétera)?; ¿qué habilidades académicas consideras has desarrollado en tu estancia en la LEI?; y sugerencias para mejorar el programa de estudios de la LEI, Plan 2011, y de otros servicios de la UPN.

En este artículo abordamos el análisis de la segunda pregunta. Respondieron alumnos entre los 18 y 33 años; el promedio fue de 24 años; 20 mujeres y 14 hombres y la mayor parte solteros (28). La mayoría proviene del sur de México (Oaxaca 12, Chiapas 11, Guerrero 3), mientras que los demás son de la región metropolitana y estados aledaños (Ciudad de México 4, Morelos 2, Hidalgo 1 y Puebla 1). Una gran parte es hablante de lengua indígena (ver tabla). 


\begin{tabular}{|c|c|c|c|}
\hline \multicolumn{3}{|c|}{ Tabla. Hablantes de lenguas indígenas } & \multirow[b]{2}{*}{ Total } \\
\hline Lengua & Primera lengua & Segunda lengua & \\
\hline Náhuatl & 4 & $3^{*}$ & 7 \\
\hline Mixe & 5 & & 5 \\
\hline Tseltal & 4 & 1 & 5 \\
\hline Mixteco & 2 & 2 & 4 \\
\hline Cho'l & 2 & & 2 \\
\hline Chatino & 1 & & 1 \\
\hline Hñahñú & 1 & & 1 \\
\hline Mazateco & 1 & & 1 \\
\hline Tsotsil & 1 & & 1 \\
\hline \multirow[t]{2}{*}{ Zoque } & 1 & & 1 \\
\hline & 22 & 6 & 28 \\
\hline * Un estu & $\begin{array}{c}\text { nte señaló que es } \\
\text { lengua. }\end{array}$ & aprendiendo la & \\
\hline
\end{tabular}

La segunda estrategia para recopilar información fue a partir de la asesoría y el acompañamiento a estudiantes durante las clases y en la elaboración de sus trabajos de titulación. Esto, en el marco de un proceso de reflexión que de forma colegiada realizamos a fin de comprender los procesos formativos y nuestras prácticas académicas.

El análisis de los datos empíricos se basó en el análisis de contenido, de acuerdo con Navarro y Díaz (1995), en un intento de recuperar el sentido que subyace a los actos comunicativos que los estudiantes expresan en sus respuestas y que van más allá de la superficie textual de los textos producidos. Procedimos a leer de forma sistemática los textos y consideramos la formación como un eje transversal del análisis; a partir de ello, las categorías se fueron agrupando en tres temas: adscripción comunitaria, revaloración y proyectos educativos y comunidad.

\section{RECONFIGURACIÓN PERSONAL Y COLECTIVA EN Y DE LOS PROCESOS FORMATIVOS}

En este apartado retomamos para el análisis las respuestas de los estudiantes a la pregunta dos del cuestionario: “CCómo ha incidido tu formación en la LEI para fortalecer diversos procesos culturales, lingüísticos, identitarios, así como la posibilidad de participar en proyectos en los que puedes desarrollar tus habilidades profesionales (en la comunidad, en distintas instituciones, etcétera)", además de los registros de acompañamiento al trabajo de asesoría que realizamos las autoras del texto.

Debemos resaltar que si bien la pregunta anterior remite a las dimensiones de lengua, cultura e identidad (en su primer parte), las respuestas de los estudiantes no siempre retoman estas categorías de modo separado. En algunas de las respuestas observamos que recuperan literalmente esas categorías, pero otros retoman perspectivas más "integrales" de lo que implica el fortalecimiento de procesos personales y sus vínculos comunitarios al reconocer, recuperar y revalorar sus relaciones con sus comunidades, saberes, lenguas indígenas y prácticas. Por ello, el análisis no se reduce a señalar cómo la LEI contribuyó a fortalecer la lengua o la cultura, o la identidad, como dimensiones separadas, sino que -y con base en las respuestas y registros- se propone una interpretación que busca ir más allá de estas categorías que, en forma tradicional, han sido y siguen siendo utilizadas para definir una diferencia, en este caso de estudiantes indígenas. 
Al mismo tiempo, desde este eje analítico de reconfiguración personal y colectiva, remitimos tanto lo que sucede con los estudiantes en los procesos formativos como lo relativo de estos procesos. Con base en la idea en, aludimos a la manera en que la experiencia formativa en el programa de la LEI ha reorientado modos de pensar y pensarse a sí mismos y a sus comunidades, así como a la relación con los otros; mientras que con la preposición de hacemos mención de las implicaciones que este proceso formativo tiene respecto de las interacciones con los docentes, la institución y sus comunidades, considerando el desarrollo de posibles intervenciones de proyectos en ellas.

\section{Formación y adscripción a las comunidades}

Los procesos formativos a lo largo de la licenciatura, tanto curriculares como extracurriculares, ofrecen a los estudiantes diversas posibilidades de acercamiento y reconocimiento con sus comunidades de origen. En intercambios con varios egresados, hemos apreciado que se produce, según comentan, un reencuentro con sus comunidades. En algunos casos, este los conduce a regresar físicamente a vivir en la comunidad; en otros, los egresados se insertan en contextos distintos y varios se quedan en la Ciudad de México. Sin embargo, cualquiera que sea el lugar en el que decidan residir una vez concluida la licenciatura, hemos advertido que muchos egresados vigorizan las relaciones con sus comunidades de origen y que su desempeño profesional, en muchas ocasiones, se orienta al fortalecimiento de los derechos y proyectos de los pueblos indígenas.

En relación con sus procesos formativos, los estudiantes expresan que la licenciatura ha contribuido de manera importante al reconocimiento de la adscripción con sus comunidades. Si bien este aspecto es abordado como temática en diversas asignaturas de la LEI, reconocen que esta se produce en forma significativa mediante el encuentro e interacción con sus pares desde su ingreso a la universidad: "Compartir experiencias con sus compañeros y compañeras me han abierto los ojos en el sentido que algunas comunidades tienen muy valorizado su lengua materna, sus fiestas patronales o comunales, sus saberes ancestrales y las relación con sus sabios (abuelos, chamanes entre otros)" (E17).

De este modo, la institución les ofrece un espacio que les posibilita ir reconstituyendo, por medio de las interacciones, una forma de vivir y de ser, así como de estar vinculados a sus comunidades de origen. En este sentido, en varias respuestas del cuestionario, como también lo hemos identificado en el aula, los estudiantes refieren un proceso formativo que toca sus maneras de pensar y actuar: "Esta carrera me ha ayudado a abrir mi mente y a emanciparme en diversos temas que me corresponden, y a crear mis propios proyectos para llevarlos a cabo sobre todo en la comunidad, en caso de las instituciones, formar parte del proyecto para llevarlo a cabo" (E22b).

La institución se convierte en un espacio donde pueden expresarse formas de significación de sí mismos, como sujetos individuales y colectivos, que han sido silenciadas u opacadas en sus experiencias de vida y escolares. En ese sentido, apreciamos procesos de reflexión que conducen a otros modos de pensar y pensarse, de una manera cercana a lo que algunos autores llaman decolonialidad. Según Walsh (2009), se trata de "un trabajo que procura a desafiar y derribar las estructuras sociales, políticas y 
epistémicas de la colonialidad -estructuras hasta ahora permanentes- que mantienen patrones de poder enraizados en la racialización, en el conocimiento eurocéntrico y en la inferiorización de algunos seres como menos humanos" (p. 12).

En el contexto mexicano seguimos apreciando, tanto en el discurso educativo como en otros espacios sociales e institucionales, la presencia, de forma velada o directa, de excluir o discriminar el "ser indígena". Esto revela la existencia de formas sedimentadas de poder, heredadas sobre todo de las políticas de construcción nacional sustentadas en propuestas educativas en las que la castellanización y la integración de las comunidades (diversas) en una identidad particular construye un relato desde la visión de los conquistadores sobre los indígenas derrotados (Pinkasz, 2011), que se superpone a la diversidad de modos de vivir y actuar en el mundo.

Los alumnos nos muestran cómo van incorporando estas formas como alejamiento de sus prácticas culturales e incluso como enojo, según podemos observar en la siguiente expresión: "Cuando entré en la carrera, el ser indígena lo veía como algo ajeno, separado a mí, veía o reconocía a mis abuelos como 'indígenas', pero [no] a mis padres, ni a mí misma. Cuando comienzo a avanzar dentro de la carrera me hace un poco más consciente de mi identidad" (E13a).

Estos discursos y significados que contradecían o socavaban su relación con saberes y prácticas sociocomunitarias han podido ser trabajadas y contestadas por los estudiantes:

He dado un gran cambio en mi forma de pensar, siento más presente mis raíces como pueblo indígena, siento la necesidad de poder contribuir en su fortalecimiento, porque lo que se vive en una comunidad es único y espiritual, el contacto con su alrededor te hace ser parte de él, su forma de comunicación que en este caso [es] mi lengua náhuatl es tan importante para seguir fortaleciendo mi cultura y nuestra identidad (E16a).

Distinguimos, en los párrafos anteriores, el peso que va adquiriendo en su proceso formativo el reencuentro con su adscripción comunitaria, la cual se hace presente a partir del desprendimiento de ideas que, producto de efectos coloniales, los alejaba y distanciaba de sus identificaciones como parte de pueblos originarios y de las historias truncadas y trastocadas en los procesos de colonización.

\section{La revaloración de sí mismos como sujetos individuales y colectivos}

Al poder reflexionar y cuestionar su propia historia escolar, así como al abordar, por ejemplo, el análisis de las políticas educativas y sus diferentes propuestas para la población indígena, en diversos cursos de la LEI se generan condiciones en las que los estudiantes recuperan de forma positiva su identidad, al reconocerse como mazatecos, chatinos, tsotsiles, entre otras adscripciones. Esto ocurre en un primer momento desde el plano individual, a partir de procesos de introspección sobre su historia, como estudiantes, como hablantes de lengua indígena, como miembros de una comunidad: "[La LEI] ha incidido en valorar aún más las diferentes culturas, las lenguas indígenas y por su puesto en cómo yo me identifico como originaria de una comunidad indígena y sobre la importancia de seguir preservando, valorando y fortaleciendo las costumbres, tradiciones y la lengua" (E25). 
En ello juegan un papel importante los procesos de interacción con sus pares. Esto se da, por un lado, con estudiantes que son hablantes de sus propias lenguas o que pertenecen a sus mismas comunidades o regiones: mixes con mixes, tseltales con tseltales, nahuas con nahuas y, por el otro, en las interacciones con los "otrosnosotros", es decir, con estudiantes que, aun cuando no hablan la misma lengua originaria o que provengan de otros pueblos, comparten historias comunes, muchas de las cuales son historias de exclusión y discriminación: "Fortalecer diversos procesos culturales, lingüísticos e identitarios. La licenciatura ha incidido mucho en estos aspectos, sobre todo a valorar todos esos conocimientos que son un legado de nuestros ancestros, y tomarlos en cuenta. También a auto-reconocerme como hablante originaria de un lugar, sin sentirme menos ante los demás" (E22).

Entonces, a partir de lo que es común, que es la historia escolar y la de sus comunidades frente a los impulsos de homogeneización cultural y negación del mundo indígena, los estudiantes van recuperando y reconstruyendo sus historias con base en formas culturales y fragmentos de historias superpuestas y subsumidas. Gracias a este proceso, formativo y de reconstrucción comprensiva, como un hermeneuta, los estudiantes pueden ir hilando y tejiendo una nueva manera de pensarse, de reconocerse a sí mismos, que es también, en parte, un viejo modo de pensarse y conocerse, desde sus vínculos con sus comunidades: "En la licenciatura me ha ayudado a ver de otra forma los aspectos culturales, en el fortalecimiento de las lenguas o lingüísticos, hay una aceptación identitaria y reconocimiento de ella" (E4a).

En este proceso, de revalorización y autorreconocimiento de sí mismos, como sujetos individuales y colectivos, los estudiantes van reconstruyendo su capacidad como sujetos ético-políticos. Nos referimos con ello a que se produce una apropiación y reapropiación de su capacidad de actuar como estudiantes universitarios y, sobre todo, como miembros de sus colectividades (Coronado, 2016). Así, encontramos entre los participantes de este trabajo estudiantes que señalan que la LEI les ha aportado una nueva forma de relacionarse con el contexto educativo y sociocultural de sus comunidades: "Mi formación ha influido primeramente en identificarme como indígena, en reconocer la importancia de la revitalización de la lengua y en considerar la diversidad cultural que existe. En lo personal este ha sido muy importante porque mi campo de trabajo es en la docencia y estos tres puntos son muy importantes en el contexto educativo" (E24).

Como en toda propuesta de estudios profesionales, destaca la importancia de que contribuya a la formación profesional y personal de los estudiantes, y también al fortalecimiento de sus comunidades de procedencia. La licenciatura permitió a varios estudiantes cambiar la idea -que tenían incorporada- de que la educación escolarizada se realizaba con marcas jerárquicas del maestro que sabe y el alumno que no. Esta transformación en su percepción del proceso educativo ocurre en la medida en que les es posible experimentar en el aula universitaria otras formas de aprendizaje y de vinculación con sus docentes y sus pares. Ocupa un lugar importante la demanda que sus profesores plantean de que participen en la construcción de conocimientos, y recuperen las experiencias y aprendizajes comunitarios.

Estas nuevas formas de posicionarse en el aula y ante su proceso educativo, así como la significativa aportación de las interacciones formativas con sus pares, han contribuido a que los estudiantes participen de manera activa durante su formación. 
Esto ha sido posible en virtud de que los conocimientos que se ponen en juego en su proceso formativo no solo tocan a aquellos planteados en la propuesta curricular, sino también a aquellos vinculados al reconocimiento de sí mismos como sujetos individuales y colectivos. Sin embargo, este proceso no ocurre lejos de tensiones, como apreciamos en el siguiente relato:

Personalmente considero que ha incidido de manera favorable en todos estos aspectos. En primer lugar porque estaba un poco peleada con mi cultura por razones de discriminación y pobreza, en segundo conocía muy poco de ella y, en tercer lugar, porque me sentía ajena a la cultura. Actualmente el proceso de formación me ha ayudado a reconciliarme con mi pasado y dejar en a un lado el dolor que causó la discriminación y las necesidades económicas. Las investigaciones me han ayudado a conocer el origen de la cultura y a valorar toda la riqueza que no había visto y mucho menos me había identificado y finalmente me siento identificada, "he encontrado mi lugar" (E30).

En la medida en que los estudiantes van avanzando en sus estudios, se analizan y discuten diversos temas que tocan procesos históricos, sociales, culturales y educativos de los pueblos indígenas, tanto del país como de Latinoamérica. Sin embargo, como hemos podido observar, en la recuperación de la experiencia en el aula y en algunas respuestas del cuestionario se presenta un conflicto cuando, desde una posición externa, los maestros, o el mismo programa, parecieran insistir en temas ante los que, como observamos en la cita anterior, no siempre se problematizan o incluso se negaban. Esta tensión, puede ser resuelta en un proceso en el que es posible vincular lo aprendido en referencia a sus comunidades. Lograr este vínculo les permite apropiarse del sentido del "nosotros" para pensar en la posibilidad de desarrollar propuestas para sus comunidades:

A través de distintos trabajos y experiencias que se han trabajado a lo largo de la carrera se ha abierto un panorama más amplio. Sobre distintas lenguas, culturas, tradiciones, etc., en los cuales se han planteado la importancia de todas y cada una de ellas, lo que como estudiantes nos hace reflexionar sobre la importancia de portar nuestra identidad y seguir transmitiendo a futuras generaciones (E12).

Otros estudiantes señalan que valoraron sus lenguas y culturas, y que ello ha contribuido, en ciertos casos, a lograr una ruptura con este proceso anclado en la desvaloración y borramiento de los pueblos, que, a lo largo de los trayectos formativos previos, los estudiantes tenían internalizado, como hemos dicho, producto de un proceso educativo castellanizador, con residuos de prácticas históricas homogeneizadoras. De ahí que no nos sorprende que un estudiante refiriera su sorpresa de que en la licenciatura pudiera vivir una experiencia formativa distinta: "Cada día nos están ayudando a nuestra formación: Pues en la revitalización de las lenguas, fue algo que yo no esperaba que sucediera, y convivencia que tengo con las diferentes culturas, costumbres, etcétera" (E11).

Tal vez, en este retorno reflexivo hacia el pasado y presente de sus pueblos originarios, que se promueve y origina en las interacciones formativas, podemos distinguir que se produce un giro del pensamiento y de estar en el mundo.

\section{Formación, proyectos educativos y comunidad}

Otro aspecto que consideramos importante ubicar en la agenda de educación superior es señalar y reconocer historias de desigualdad y de inequidad en procesos de formación previos a su inserción universitaria. De igual modo, nos interesa resaltar, 
entre otras tensiones, la interacción entre diferentes saberes y prácticas que tenemos los docentes y los estudiantes, y reconocer que los procesos formativos se desarrollan en el marco de relaciones interculturales marcadas por la desigualdad y las relaciones de poder imperantes en instituciones de educación superior.

Entre estas tensiones, identificamos algunas relacionadas con imágenes/valoraciones que los estudiantes tienen de sus profesores, es decir, de nosotras/os como formadores respecto a cómo enseñamos, en qué aspectos hacemos hincapié, qué esperan de nosotros, y si están presentes (o no) obstáculos en la interacción con los docentes que pueden dificultar un posicionamiento activo en su proceso formativo.

Las interacciones con los maestros adquieren una complejidad a la hora en que nos planteamos establecer un diálogo académico, reconociendo nuestras diferencias, sobre todo en el origen de los formadores, muchos de nosotros no indígenas, y de los estudiantes, en su mayoría indígenas. En este sentido, resulta relevante considerar la relación con nuestros estudiantes como de acompañamiento (Navia, 2006).

El programa les ofrece un espacio en el que se pueden posicionar como sujetos de su propio aprendizaje, así como expresarse, recuperar su experiencia y utilizar lo aprendido para dar sentido a sus aprendizajes previos (Magnan, 1997). Distinguimos una relación de colaboración y ayuda centrada en los estudiantes, que favorece el intercambio de saberes y la apertura de espacios de reflexión. No obstante, existen retos aún por atender vinculados a esta temática, y como algunos estudiantes han señalado, es necesario dedicar más actividades orientadas a la formación práctica para "poner a prueba la teoría" (E12b).

Los estudiantes reconocen que durante sus estudios van construyendo conocimientos y disposiciones favorables para impulsar proyectos en y para sus comunidades. Sobre el primer punto, observamos un proceso de interacción con sus formadores y con sus pares, y también un proceso reflexivo de conocimiento, redescubrimiento y autorreconocimiento de diversas culturas y lenguas, así como la propia. Este proceso que es formativo, en el sentido que los toca a sí mismos, en un giro de sus formas de pensar y pensarse, produce en los estudiantes un deseo de dar a conocer a los otros y a los miembros de sus comunidades lo aprendido, lo que se traduce en posibilidades de intervención y desarrollo de proyectos.

Esto sucede porque valoran la utilidad de los conocimientos y las habilidades desarrollados en el programa, entre ellos diseñar proyectos y estrategias, adecuar programas educativos de acuerdo con las necesidades de la población y realizar diagnósticos:

Tenemos el compromiso con las comunidades que han sido el foco central en los 4 años de carrera, a través de ella nos formamos y nos queda fortalecer la cultura en toda la extensión de la palabra, no es imposible puesto que en lo personal me ayudó conocer y reconocer lo importante que son los procesos culturales, darle voz a lo que se ha estado perdiendo pero sobre todo tomar conciencia de lo importante que son los pueblos originarios, sus costumbres y una educación pertinente para ellos (E29).

Se distingue en varias respuestas cómo se va configurando una disposición para actuar con sus comunidades con expresiones como "busco poder trabajar en proyectos comunitarios y educativos" (E13) y "la LEI me ha incidido a que yo como ciudadano regrese a mi comunidad para hacer los trabajos comunales que se respetan y valoran dentro de la comunidad, este con la mentalidad de poder apoyar nuestras raíces y costumbres" (E28). 
Existe, a su vez, un reconocimiento de las posibilidades de intervenir en la formación y reconfiguración de la adscripción a sus comunidades, y reconocer que pueden contribuir a procesos que reviertan la pérdida y el abandono de sus formas de vivir o, en su caso, las fortalezcan, como distinguimos en estas respuestas: "aprendí que la palabra o voz de los abuelos como portadores de cúmulo de conocimiento se puede conservar" (17a) y "podemos realizar intervenciones para fortalecer y mantener la identidad de un grupo social" (E18).

Entre las modalidades de intervención, se mencionaron el realizar talleres; proyectar "videos de otra cultura indígena en donde exprese la importancia de la cultura y nuestra forma de vivir" (17a); integrar la educación comunitaria y la escolar para "reforzar la lengua y los diferentes saberes que dan identidad a la comunidad [...] e inducir en proyectos el currículum y su pertinencia en la cultura" (E32); proponer estrategias de trabajo, aportar escritos, traducciones a instituciones, producción de textos; identificar intereses y necesidades de las comunidades, repensar los procesos educativos en la comunidad, así como los procesos lingüísticos, entre otras.

Sin embargo, los estudiantes reconocen que es necesario ampliar más la dimensión práctica, lo que incluye tanto procesos impulsados por los profesores como también por sí mismos. Atender lo anterior implicaría una revisión del peso que tiene en la academia "tradicional" la formación teórica y su articulación con el desarrollo de habilidades y prácticas de intervención con los estudiantes.

\section{A MANERA DE CONCLUSIONES}

Los procesos formativos en programas educativos como la LEI se convierten en espacios que permiten pensar y reformular lo que en el campo de la educación para pueblos indígenas se ha caracterizado con nociones como "relaciones interculturales", "diálogo de saberes" o formas de "colaboración intercultural".

Los estudiantes resaltan diferentes aspectos que han fortalecido su proceso académico y, en ese camino, se reconfiguran procesos identitarios individuales y colectivos, aunque no sin tensiones, marcados por condiciones diversas de vinculación e identificación con sus familias/comunidades.

Un sector del alumnado, al estar concluyendo sus estudios, expresan una especie de reencuentro con sus pueblos y comunidades de origen, revaloran sus lenguas y culturas, y construyen perspectivas nuevas, así como también, en ocasiones, critican los procesos educativos escolarizados para niñas, niños y jóvenes indígenas.

Debemos resaltar que, si bien la revaloración del proceso formativo experimentado en la LEI tiene en sus respuestas la liga a nociones de fortalecimiento de sus culturas, lenguas (indígenas) e identidad(es) -aspecto que se indicaba en la pregunta del cuestionario-, la visión sobre este fortalecimiento se da en términos más complejos que el solo hecho de afirmar "sí, en el programa fortalecí mi lengua o mi cultura o mi identidad". Los testimonios más bien dan cuenta de actos de reflexión sobre sí mismos, sus colectivos y sobre "otros", en los que se repiensan como sujetos activos más allá de lo que en el discurso intercultural o neoindigenista se quiere marcar con "recuperar la lengua, la cultura y la identidad". En este 
sentido, nos muestran maneras otras de pensar (Walsh, 2009), comprender y conformar procesos de subjetivación y revisión de sus historias socioculturales.

Desde un punto de vista reflexivo, como formadoras de profesionales indígenas en el campo de la educación, consideramos que la experiencia universitaria en esta licenciatura fortalece posiciones y subjetividades en los estudiantes. Lo anterior se relaciona, en parte, con procesos de decolonialidad, cuando reconocemos que los estudiantes deconstruyen sus maneras de pensar y pensarse, y con procesos descolonizadores, entendiendo por ello en este contexto educativo la condición de posibilidad para un análisis crítico de las "historiografías" descentradas y de las nuevas relaciones de poder (Mateo, 2009). En este escenario también es importante indicar, por ejemplo, el hecho de que más del 50\% del estudiantado en el programa son mujeres indígenas, lo que es significativo en términos de que para ellas el acceso a la educación superior ha implicado rupturas y transformaciones con los mandatos de género que prevalecen en muchas comunidades, pero que, al mismo tiempo, les abre otras oportunidades para sí mismas y para sus colectivos (Czarny, 2012).

Coincidimos con Mato (2017) en que en las instituciones de educación superior que incorporan programas y población, en este caso indígena, se trata más bien de avanzar en un "hacer juntos", en la "construcción de modalidades duraderas y mutuamente provechosas de colaboración intercultural” (p. 13). Sin embargo, en el contexto de la educación superior, encontramos que prevalecen significados y prácticas que aún revelan formas de exclusión y discriminación que impiden una atención educativa justa y equitativa a los jóvenes indígenas que ingresan a las universidades. La tan difundida política de reconocimiento de la diversidad, la multiculturalidad, el respeto a las lenguas, entre otros discursos, quedan aún como tareas pendientes en nuestras instituciones.

Del mismo modo vale replantearse la imposición de formas académicas eurocentradas, que obturan la posibilidad de reconocer que existen otros conocimientos y otras formas de producir y transmitir el conocimiento, u otras formas de pensar y concebir a las universidades desde una visión interintracultural con sentido crítico (Prada y López, 2009). De ahí la relevancia de reflexionar y repensar nuestras prácticas formativas y las relaciones intersubjetivas que se hacen presentes en los procesos de formación.

\section{REFERENCIAS BIBLIOGRÁFICAS}

Arendt, H. (2001). ¿Qué es la política? Barcelona: Ediciones Paidós Ibérica /Instituto de Ciencias de Educación de la Universidad Autónoma de Barcelona.

Coronado, M. (2016). La cultura etnomagisterial en Oaxaca. Oaxaca: UPN 201 Oaxaca.

Czarny, G. (coord.) (2012). Jóvenes indígenas en la UPN. Relatos escolares desde la educación superior. México: UPN.

Dietz, G. y Mateos, L. (2010, enero-junio). La etnografía reflexiva en el acompañamiento de procesos de interculturalidad educativa: un ejemplo veracruzano. Cuicuilco, vol. 17, núm. 48, pp. 107-131. México: ENAH.

Magnan, M. (1997). Accompagner les parcours de formation. Lyon: CAFOC de Lyon. 
Mateo, J. (2009, junio). La condición "postcolonial": identidades dinámicas entre la diferencia y el sincretismo. Relaciones Internacionales, núm. 11, pp.191-197.

Mato, D. (2017). Del "diálogo de saberes" a la construcción de modalidades de "colaboración intercultural": aprendizajes y articulaciones más allá de la academia. Lasa/Oxfam, vol. XLVIII, núm. 3, pp. 8-17.

Mignolo, W. (2010). Desobediencia epistémica (II). Pensamiento independiente y libertad de-colonial. Otros logos. Revista de estudios críticos, núm. 1, pp. 8-42.

Navarro, P. y Díaz, C. (1995). Análisis de contenido. En J. M. Delgado y J. Gutiérrez (coords.). Métodos y técnicas cualitativas de investigación en ciencias sociales (pp. 177-224). España: Editorial Síntesis.

Navia. C. (2006). Autoformación de maestros en los márgenes del sistema educativo. Cultura, experiencia e interacción formativa. México: Pomares.

Pinkasz, D. (2011). Continuidades y rupturas en la escuela y el currículum de la modernidad. En S. Finocchio y N. Romero (comps.). Saberes y prácticas escolares (pp. 17-39). Rosario: Homo Sapiens Ediciones.

Prada, F. y López, L. E. (2009). Educación superior y descentramiento epistemológico. En L. López (coord.). Interculturalidad, educación y ciudadanía. Perspectivas latinoamericanas. Bolivia: FunProeib Andes-Plural.

Restrepo, E. y Rojas, A. (2010). Inflexión colonial: fuentes conceptos y cuestionamientos. Cauca: Editorial Universidad de Cauca.

Ricoeur, P. (2001). Del texto a la acción. Ensayos de hermenéutica II. México: Fondo de Cultura Económica.

Salinas, G. (2011). Formar docentes para la educación indígena: un acercamiento antropológico a una experiencia universitaria. Tesis de maestría. Escuela Nacional de Antropología e Historia. México.

Sampieri, R. (2006). Metodología de la investigación. México: McGraw-Hill.

Suárez, D. (2010). Docentes, narrativa e investigación educativa. La documentación narrativa de las prácticas docentes y la indagación pedagógica del mundo y las experiencias escolares. En I. Sverdlick (comp.). La investigación educativa. Una herramienta de conocimiento y de acción. Buenos Aires: Noveduc.

Tubino, F. (2007). Las ambivalencias de las acciones afirmativas. En J. Ansion y F. Tubino (eds.). Educar en ciudadanía intercultural. Experiencias y retos en la formación de estudiantes universitarios indígenas (pp. 91-110). Lima: Fondo Editorial de la Pontificia Universidad Católica del Perú.

Vasilachis, I. (2007). La investigación cualitativa. En I. Vasilachis (coord.). Estrategias de investigación cualitativa (pp. 23-65). Buenos Aires: Gedisa.

Villa, L. (2018, 9 de agosto). Indígenas, sólo el 1\% de la matrícula universitaria en México. Notiamérica. México. Recuperado de https://www.iis.unam.mx/ blog/wp-content/uploads/2018/08/35.pdf

Walsh, Catherine. (2009). Interculturalidad crítica y pedagogía de-colonial: insurgir, re-existir y re-vivir. En P. Medina (coord.). Educación intercultural en América Latina. Memorias, horizontes históricos y disyuntivas políticas (pp. 27-54). México: Universidad Pedagógica Nacional/Conacyt/Plaza y Valdés. 